\title{
INDEX TO VOLUME III.
}

Acanthaclisis, 40. fundatus, 44 . subtendeus, 44.

Acronycta hasta, 116. hastulifera, 116. leporina, 116. oblinita, 116. occidentalis, 116.

Adela, 123. degeerella, 124.

Aegeriidae, 100, 122.

Alsophila pometaria, 113.

Amendments passed, 8.

Amphion nessus, 109.

Amphorophora, 320. ampullata, 317, 320

Anarsia lineatella, 23.

Angoumois grain moth, host of Pediculoides, 19

Anisota, 110.

Anosia plexippus, 106.

Anthomyia, 287.

Anthonomus eugenii, 23. grandis, 23.

Anthophora retusa, 20, 22.

Anuraphis, 314.

Apantesis parthenice, 119.

Apatelodes, 109, 124. angelica, 109 . torrefacta, 109.

Aphidinae, 314.

Aphidini, 321.

Aphis, 314, 315. pyri, 314

sambuci, 314

tormentillae, 314 . ulmi, 315 .

Aplodes sp., 113.

Apparatus for the Determination of Optimums of Temperature and Moisture, 147.

Aranea, 173, 175. circulata, $176,177,179,181$. frondosa, 173, 177, 179, 181. gigas, 179 . ocellata, 177

Araneinae, 175

Arctiidae, 99. 118.

Arctiinae, 118.

Argynnis cybele, 106.

Argyresthia goedartella, 124.

Artace pemectistriga, 110.

Assets, 4.

Athysanini, 215.

Atypus bicolor, 168.

Banks, N., article by, 40 .
Basilarchia disippus, 106.

Basilona, 110

Bombycia or, 112.

Bombycidae, 107, 111, 124.

Bombyx mori, 111.

Boston meeting, Minutes, 1.

Botys polygonalis, 124.

Brachycolus, stellariae,

Bradley, C. J., title of paper, 9.

Brephos nothum, 113

Cacoecia, 122.

Cacoecia cerasivorana, 124.

California Rodent Fleas, Bionomics of, 61 .

Calliphora, 287.

Callipodidae, 233.

Callistoleon, 40, 42 .

erythrocephalum, 42 .

Calocampa, curvimacula, 116.

Cambalidae, 258.

Carpocapsa pomone!1a, 124.

Caseya irritans, 241.

Cash receipts, 4 .

Caterpillars, structure of, 94 .

Catocala cara, 117.

Catocalinae, 117.

Cecidomyia resinicola, 48.

Cecidomyia resinicoloides, Anatomy of larva, 45 .

Ceramica picta, 117 .

Ceratophyllus acutus, $61,62,63,65,67$. $69,71,76$.

canis, 70 .

fasciatus, $63,70,76,81,82$. musculi, 70 .

Cerosipha, 322,323 . passerinina, 323

Ceratomyia amyntor, 108 .

Cercyonis alope, 106.

Cerura, 112

Chamberlin, R. V., article by, 233.

Chauliodes, 281.

Chermes ulmi, 315 .

Chigger, reference to, 15 .

Chironomus, 287, 288.

Cilix glaucata, 113.

Citheronia, 110.

Chrysoleon, 40, 43 . punctatum, 43.

Clisiocampa, 286, 294.

Cicada septendecem, 23.

Cnidocampa flavescens, 120.

Coleophora, 124.

Collembola, 277, 293, 298, 299. 
Coloradoa, $322,323$. rufomaculata, 323.

Comstock, J. H., article by, 161.

Conotyla coloradensis, 237. deseretae, 235.

Corydalis, 280. cornuta, 281

Cosmopteryx scribaiella, 124.

Cossidae, 121.

Cossus cossus, 122 .

Cosymbia lumenaria, 113.

Crambus falsellus, 124

Craspedosomidae, 235.

Crosby, C. R., title of paper, 9.

Cryptosiphum, 322, 325 . artemisae, 325.

Ctenucha virginica, 119.

Ctenophora, 287.

Cucullia, 116.

Culex, 281, 304.

Cybister, 293.

Darapsa myron, 109.

Datana integerrima, 112. ministra, 112 .

Davis, J. J., title of paper, 9.

Deilephila gallii, 109 .

Deltocephalinae, 215.

Deltocephalus . 214, 215.

Depressaria, 122, 123. putridella, 124.

Demas coryli, 115.

Daicrisia virginica, 119.

Diapheromera femorata, structure of the egg, and significance of resemblance of eggs to seeds, 83 .

Diplopoda from the Western States, 233

Distoleon, 40, 42.

bistrigatus, 43. verticalis, 42 .

Ditropinotus aureoviridis, 37.

Dolomedes fontanus, $181,182$.

Dorydini, 205.

Drasteria crassiuscula, 117. erechtea, 117.

Drepanidae, 101, 112.

Drepana arcuata, 113. falcataria, 113.

Dolba hylaeus, 109.

Dysdera, 167. interrita, 167.

Earias chlorana, 116.

Edwards, W. H., Resolutions on Death of, 10

Elrod, M. J., title of paper, 9.

Endromidae, 111.

Endromis versicolor, 111.

Endrosis, 122, 123.

lacteella, 124.

Estigmene acraea, 119.
Eubaphe nigricans, 118.

Euchaetias egle, 118.

Euclea delphinii, 120.

Eucleidae, 120.

Euphoeades troilus, 105.

Euphydryas phaeton, 106.

Euploeinae, 106.

Euproctis chrysorrhea, 115

Eutettix, 215.

Euvanessa antiopa, 106.

Ewing, H. E., article by, 209.

Executive Committee Report, 2.

Expenditures classified, 3.

Feltia, 117.

Fidonia truncataria, 113.

Field, W. L. W., title of paper, 6.

Filistata, hibernalis, 162 .

Fleas, California Rodent, Bionomics of, 61 .

trapping of, 66 .

Fontaria tuobita, 243.

Forbes, W. T. M., article by, 94. title of paper, 9 .

Formicaleo, 40. dispersus, 43. marginalis, 44 . septus, 44

Frenatae, 104 key to families, 102.

Galleria mellonella, 124 .

Geometridae, 100, 107, 113.

Glenurus, 40.

dissolutus, 40.

pulchellus, 40 .

stigmatus, 40 .

Gluphisia, 107.

Gracilaria, 122 alchimiella, 124

Greenidea, 316, 317 artocarpi, 317.

Gymnobates, 211.

Habrosyne derassa, 112.

Hadena turbulenta, 117.

Halesidota caryae, 118.

maculata, 118 .

tesselaris, 118 .

Hapalia indicans, 154.

Hammar, A. G., title of paper, 9.

Haseman, L., article by, 277. title of paper by, 9 .

Headlee, T. H., article by, 147. title of paper by, 9 .

Hecatera, 154.

Hemerocampa leucostigma, 115.

Hepialus, 96.

Hesperiinae, 105.

Heterocampa guttivittata, 112.

Heterogynidae, 120. 
Heterogynis paradoxa, 120.

Heteropus ventricosus, 15.

Homoeosoma, 122. nebulella, 124 .

Hoplopsyllus anomalus, 78 .

Hormaphis hamamelidis, life cycle of, 144 .

Hyadaphis, 321, 322.

Hyalopterus, 322,324 . aurantiae, 324 .

Hydria undulata, 115.

Hydrocampa nymphaeata, 124.

Hydrophilus, 293

Hydrobius, 293.

Hydrous, 293.

Hypeninae, 118.

Hypena humuli, 118.

Hyperchiria io, 111.

Hyphantria textor, 119.

Hypochilus, 169.

thorelli, 164, 169.

Idiopterus, 317,319 .

nephrelepidis, 319 .

I1linoia, $317,318$.

liriondri, 318.

Incurvaria, 123 ,

koerneriella, 124.

Ingura, 117.

Insects and Entomologists, 12.

Isia isabella, 119.

Isosoma grande, 23, 29.

hordei, 23

tritici, 30,33 .

Jasoniades glaucus, 105.

Jugatae, 104.

Kepolydesmus, 246, 247.

Lacosoma, 124.

chiridota, 114

Lacosomidae, 114, 124.

Laertias philenor, 105.

Lagoa crispata, 120.

Lasiocampidae, 107, 110, 124.

Leptotrachelus dorsalis, 23.

Lepthyphantes minuta, 183.

Libythia celtis, 105 .

Libytheidae, 100, 105.

Libytheinae, 105.

Linyphyia, 170, 172, 175. phrygiana, 170, 173.

Linyphiidae, 170.

Liosomaphis, 321, 322. berberidis, 322 .

Lithosiinae, 118.

Lithosia complana, 118

Loemopsylla cheopis, $61,70,76,81$.
Lophodonta, 112.

Loxosceles, $165,167$.

Loxosceles rufescens, 175.

Lycaenidae, 101, 105.

Lycia cognataria, 113.

Lyman, H. H., title of paper, 5.

Lymantriidae, 114.

Lysiopetalum mutans, 233.

Macchiatiella, 314.

Macgillivray, A. D., title of paper, 5, 9 .

Macrosiphini, 217.

Macrosiphum, 317, 318. rosae, 318 .

Malacosoma, americana 110. disstria, 110.

Mamestra species of, 154 . circumcincta, 158

illaudabilis, $154,155,157$.

laudabilis, $154,155,157$.

marinitincta, 157

olivacea, 158 .

restora, 156,157 .

spiculosa, 157.

stricta, 157,158 .

strigicollis, 157 .

tenisca, 158

Mastopoda, 321, 323.

pteridis, 323 .

Matheson, R., title of paper, 5 .

Megalopygidae, 120.

Melalopha, 99, 124. apicalis, 112.

Melalophinae, 112.

Melittia cucurbitae, 122.

Meromyza americana, 22.

Mesamia, 214.

Microlepidoptera, 122.

Microparsus, 317, 319. variabilis, 319 .

Microsiphon, 314.

Microsiphum, 322, 324. ptarmicae, 324

Minutes, Boston Meeting, 1.

Mirabilis jalapa, 89 .

Mite, Predaceous, noxious to man, 15.

Mitzmain, M. B., article by, 61 .

Moisture apparatus, 147.

Mordellistina ustulata, 24 .

Morgan, Miss A. H., title of paper, 5.

Morgan, T. H., and Shull, A. F., article by, 144 .

Myrmeleon, 40. croceicollis, 44 pictifrons, 44. uniseriatus, 44.

Myrmeleonidae from Australia, 40.

Myzus, 314, 318, 321.

cerasi, 321 . 
Nadata, 107

Nadata gibbosa, 112

Nectarosiphon, 317,318 rubicola, 318

Nepticula, 123. pomivorella, 124.

Noctua, 117

Noctuidae, 97, 100, 107, 115, 122

Noctuinae, 115.

Nolidae, $100,120$.

Nola cucullata, 121

Notodontidae, 101, 107, 111, 124.

Notodontinae, 112.

Nycteolinae, 117.

Nycteola revayana, 117 .

Nymphalidae, 105.

Nymphalinae, 106.

Officers elected for 1910, 6.

Opheltes glaucopterus, 186,197

Optimums of Temperature and Moisture, 147.

Oribatidae, 209.

Oripoda, 210 elongata, 211

Orneodes, 123 .

hexadactyla, 124

Oxyptilus hieracii, 124.

Pachygnatha, $169,170$.

Paeromopidae, 257.

Paeromopus lysiopetalinus, 257.

Paiteya, 258 errans, 258.

Paniscini, North American, 186.

Paniscus, alaskensis, 197.

albotarsatus, 198.

albovarigatus, 198 .

geminatus, 186, 198, 201.

medius, 198

melanostigma, 199 .

nigripectus, 199

ocellatus, 199

subfuscus, 200.

texanus, 200

tinctipennis, 201

Panopoda rufimargo, 117

Panorpa, 123.

Panorpata, 99

Pantheinae, 115.

Paonias myops, 109

Papilio polyxenes, 105

Papilionidae, 100, 105.

Papilionina, 104, 105.

Paraiulidae, 253.

Paraiulus furcifer, var. sinampus, 256. tiganus, 254

zakiwanus, 253 .

Pediculoides noxious to man, 15 . tritici, $15,16,17,19$.

yentricosus, $15,18,19,20,27$.
Pentalonia, $317,319$.

nigronervosa, 319 .

Pergandeida, 321, 323. ononidis, 323.

Phalonia, 123. alcella, 124 .

Phlegethontius celeus, 109. sextus, 109

Pholisora catullus, 105.

Pholus pandorus, 109

Phorodon, 318, 320. humuli, 320 .

Phyllium crurifolium, 86. scythe, 85.

Physogaster larvarum, 20.

Pieridae, 105.

Pieris brassicae, 105 . rapae, 105.

Pisauridae, 181

Platymetopius, Am. species of, 214. acutus, 217, 219, 221 var. dubius, 220 . angustatus, $217,223$. brevis, 218,230 .

cinereus, 217,223 .

cuprescens, $217,221$.

dorsalis, $216,224$.

elegans, 216, 219.

frontalis, 217,225 .

fuscifrons, $217,230$.

fulvus, $217,224,225$.

hyalinus, 216,218 .

irroratus, 218, 227 .

latus, $217,221$.

loricatus, 217, 226.

magadelensis, 215.

majestus, 216, 219

modestus, 230.

nanus, 217,226

nasutus, 217,225 .

nigriviridis, 216,218

var. dixianus, 218.

obscurus, 217, 227, 229.

oregonensis, 217,220 .

osborni, $218,229$.

rostratus, 214.

scriptus, $218,228$.

tenuifrons, 216,231

slossoni, $217,222$.

undatus, 214.

verecundus, $216,218,227$.

vittatus, 214.

Polydesmidae, 244.

Polydesmus amandus, 249.

anderisus, 246.

sastianus, 251.

socarnius, 252

sontus, 247.

Polygonia interrogationis, 106.

Porthetria dispar, 114. 
Protoplectron, 40. costatus, 41 . pallidum, 41.

Pseudosphinx tetrio, 108.

Psyche zelleri, 121.

Psychidae, 97, 100, 121.

Psychinae, 121.

Psychoda, 281.

Psychoda alternata, structure and metamorphosis of the alimentary canal of larva of, 277.

Pterophoridae, 123.

Ptychoptera contaminata, 277, 293.

Pulex irritans, 61, 63, 64, 65, 69, 70, 81 . Pygerinae, 112.

Pyralididae, 100, 122, 123.

Pyrophila pyramidoides, 117.

Receipts, cash, 4.

Report of Auditing Committee, 7 . Executive Committee, 2.

Committee on Resolutions, 7 .

Resolutions on death of Wm. H. Edwards, 10.

On death of M. V. Slingerland, 11.

Rhodophora, 117.

Rhopalosiphum, 318, 320.

persicae, 320 .

Rodent Fleas, Bionomics of, 61.

Sarcopsylla, penetrans, 15 .

Sarcoptides psoriques, 15.

Saturniidae, 107, 111, 124.

Saturniina, 110, 124.

Scaphoideus, 215.

Schizoneura ulmi, 315.

Schizura badia, 112.

concinna, 112.

unicornis, 112 .

Scopelosoma, 116.

Scytonotus, granulatus, 245 . piger, 244.

Sesiidae, 122.

Severin, H. H. P., article by, 83 .

Shull, A. F., and Morgan, T. H., article by, 144

Simaethis, 122 .

Simaethis oxyacantha, 124.

Simulium, 287.

Simyra henrici, 116.

Siphocoryne, 322.

Sitotroga cereale1la, 19, 29, 30, 33. 124.

Slingerland, M. V., Resolutions on death of, 11.

Smith, J. B., Abstract of Address by, 12. Article by, 154.

Solenobia, 123.

Solenobia pineti, 121.

Solenobiinae, 121

Sphingidae, 107, 108, 124.
Sphinx gordius, 108

Spiders, palpi of male, 161.

Stratiomyia, 287.

Striaria nazinta, 242.

Striariidae, 242.

Suriana maritima, 89.

Synchlora, aerata, 113.

Syntomidae, 119.

\section{Tanypus, 287.}

Telea polyphemus, 111.

Temperature, apparatus, 147.

Tenebrio molitor, 299.

Tetragnatha extensa, 169 .

Tetraneura ulmi, 315 .

Thaumetopoeidae, 114.

Thaumetopoea, 114.

Thecla ilicis, 105.

Thyatiridae, 100, 101, 107, 112.

Thyridopteryx ephemeraeformis, 121.

Thyris, 123 .

Thyris vitrina, 124 .

Tineidae, 99.

Tineina, 100 .

Tineola, 123 .

biselliella, 124.

Tingupa, 238.

monterea, 240.

utahensis, 238.

Tipula, 287.

Tortricidae, 99, 122, 123.

Toxoptera, 318,320 .

aurantiae, 320 .

Trichoptera, 99 .

Trichosiphini, 316.

Trichosiphum, 316 . aonae, 316 .

Triggerson, C. J., title of paper, 5.

Tropaea luna, 111.

Van Duzee, E. P., article by, 214.

Walking Stick, structure of egg, 83.

Washburn, title of paper, 6 .

Webster, F. M., title of paper, 5. article by, 15 .

Wheeler, W. M., title of paper, 5.

Whitmarsh, R. D., article by, 186.

Williams; F. X., article by, 45.

Wilson, H. F., article by, 314 .

Xystodesmidae, 243.

Yponomeuta, 122, 123. cagnagellus, 124.

Zerene catenaria, 113.

Zygaenidae, 120.

Zygaena trifolii, 120.

Zygaenina, 119. 


\section{$2 \mathrm{BHL}$ Biodiversity Heritage Library}

1910. "Index to Volume III." Annals of the Entomological Society of America 3 , 327-331. https://doi.org/10.1093/aesa/3.4.327.

View This Item Online: https://www.biodiversitylibrary.org/item/38713

DOI: https://doi.org/10.1093/aesa/3.4.327

Permalink: https://www.biodiversitylibrary.org/partpdf/193752

\section{Holding Institution}

Smithsonian Libraries

\section{Sponsored by}

Smithsonian

\section{Copyright \& Reuse}

Copyright Status: NOT_IN_COPYRIGHT

This document was created from content at the Biodiversity Heritage Library, the world's largest open access digital library for biodiversity literature and archives. Visit BHL at https://www.biodiversitylibrary.org. 